\title{
Localization of WSN Using Fuzzy Inference System with Optimized Membership Function by Bat Algorithm
}

\author{
Hao Shi, Wanliang Wang and Liangjin Lu \\ College of Computer Science and Technology, Zhejiang University of Technology, \\ Hangzhou 310023, China \\ shihao@inbox.com
}

\begin{abstract}
Localization is one of the most important research topics in the wireless sensor network applications. To improve the indoor localization accuracy, the centroid localization algorithm based on Mamdani fuzzy system has been adopted to attain the weight between sensor node and anchor node. This paper proposes a novel optimized input membership function by bat algorithm in fuzzy inference system using the data of received signal strength in real indoor condition. The author has realized the algorithm on Zigbee platform and the experimental comparison on other different centroid localization algorithms indicates that Mamdani fuzzy inference adopting the membership function optimized by bat algorithm renders smaller mean localization errors.
\end{abstract}

Keywords: Wireless sensor network (WSN); weighted centroid localization algorithm; fuzzy inference system; bat algorithm; received signal strength (RSS)

\section{Introduction}

Wireless Sensor Networks (WSN), as the key technology of the base network of the Internet of Things [1], has increasingly attracted researchers' attention. Considering that GPS signal can't be available indoors, the localization technology of WSN has been usually applied to the systems of indoor fire localization, safety monitoring, indoor navigation and health monitoring. In indoor localization application, as node signal of sensors is subject to the indoor obstacles and personnel movement, the localization accuracy is not that favorable. Therefore, specific algorithm should be adopted to improve the accuracy of sensor node localization [2].

Localization methods of WSN is divided into two types: range-based localization methods and range-free localization methods [3]. The range-based localization methods adopt complicate hardware to obtain the distance and angle among nodes and thus to calculate the localization information, such as TOA (time of arrival), TDOA (time difference of arrival), AOA (angle of arrival) and RSSI (received signal strength indicator) methods. The range-free localization methods calculate the distance based on the network connectivity of WSN. In spite of its deficiency in accuracy localization, it boasts simple equipment and lower cost and thus is widely applied [4], such as centroid localization, DV-Hop [5], APIT [6], and MDS-MAP [7] algorithms. In [8], based on the empirical model of signal transmission, the distance between the transmitter and the receiver can be attained by querying the mapping database. In [9], improved DV-Hop algorithm is proposed and artificial bee colony algorithm is used for estimating the localization coordinates. In [8-10], the accuracy of localization depends on the accuracy of the RSSI distance model. However, in indoor localization system application, the real transmission of radio wave differs from that in ideal spherical model, as the radio wave signals will refract, reflect, and scatter indoors and thus affect the accuracy of the estimation. In [11], fuzzy inference system (FIS) is adopted for estimating the weight between nodes and weighted centroid algorithm for the coordinates of the node. However, 
the disadvantage of the method is that the slope of triangular membership functions are the same. In real conditions, due to the differences of wireless sensor antenna, processes and real environment, RSS and the relation of RSSI and distance may not be of linear relations, for which certain localization error will be caused for the method. In this paper, to adapt to the localization in complicated indoor environment, the author has proposed a type of fuzzy weighted centroid localization algorithm based on RSS. In the algorithm, the membership functions is optimized by bat algorithm, which data come from different distances between sensor node and anchor node in real indoor environment. Then Mamdani fuzzy system gains the accuracy weight and thus get the relatively accuracy centroid coordinates, reducing the impact of radio wave in indoors, and improving the system localization accuracy.

\section{Localization Algorithm Model}

\subsection{RSS Features and Ranging Model Analysis}

Radio wave power decreases along with increasing distance. In free Space propagation model [12], signal transmission power and received power can be represented as below:

$$
\frac{P_{r}}{P_{t}}=\frac{G_{t} G_{r} \lambda^{2}}{(4 \pi d)^{2}}
$$

where $P_{r}$ is the power of received signal; $P_{t}$ is the power of transmitted signal; $G_{t}$ is the transmitted antenna gain; $G_{r}$ is the received antenna gain; $\lambda$ is the wave length; $d$ is the distance between transmitting terminal and receiving terminal. From (1), we can see the received power is square inversely proportional to the distance. In the application of wireless sensor network application, distance can be calculated from the received signal strength, which can be showed in the following equation:

$$
P_{l}(d)=P_{l}\left(d_{0}\right)-10 \times n \times \log \left(\frac{d}{d_{0}}\right)+X_{p}
$$

where $P_{l}(d)$ is the received signal power at the distance of $d ; n$ is the path loss coefficient varying based on antenna and environment and usually between 2 and $4 ; d$ is the distance between the transmitting and receiving terminals; $d_{0}$ is the reference distance which is usually one meter; $X_{p}$ is Gaussian distribution random variable with mean value of zero which stands for the impact of environment factor on transmission power. In Zigbee protocol, each data frame contains the RSS strength value. Thus, signal strength can be obtained by reading the data frame. This method requires no extra hardware and only reading data frame is sufficient for obtaining the signal strength.

In real indoors, the RSS of received power of radio frequency chip is not of linear relation to distance. Fix the transmitting and receiving terminals on platforms one meter from the ground, set the node transmitted power at $0 \mathrm{dBm}$ and adjust the distance between the transmitting and receiving terminals. Measure the relation between RSS and distance and separately calculate the mean value and standard deviation of RSS at different distances. In real environment, due to the difference of electronic components of sensor nodes and processes, the RSS value between nodes doesn't simply decrease along with the increasing distance between nodes.

\subsection{Weighted Centroid Localization Algorithm}

Centroid algorithm is a type of range-free localization algorithm, using neighboring anchor nodes' coordinates to calculate the coordinates of unknown sensor nodes as shown in Figure 1. 


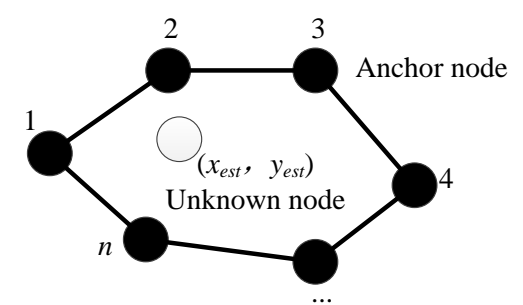

Figure 1. Schematic Diagram of Centroid Localization Algorithm

As shown in Figure 1, the unknown node $\left(x_{e s t}, y_{e s t}\right)$ is surrounded by $n$ anchor nodes which broadcasts their localization information. After having received the position information of the anchor nodes, the unknown node starts to calculate its own coordinates in the formula as below:

$$
\left(x_{e s t}, y_{\text {est }}\right)=\left(\frac{\sum_{i=0}^{n} x_{i}}{n}, \frac{\sum_{i=0}^{n} y_{i}}{n}\right)
$$

where $\left(x_{e s t}, y_{e s t}\right)$ represents the estimated coordinates of the unknown node, $\left(x_{i}, y_{i}\right)$ indicates that of the anchor nodes, $n$ is the number of the anchor nodes surrounding the unknown node. The higher the $n$ is, the smaller the error of the localization error of the unknown node. In spite of the simple method, as the sensor nodes are constrained by energy and others, $n$ is usually of a small number which results in larger localization error and fails to meet the requirements of real conditions usually. To improve the localization accuracy, weighted centroid localization algorithm is adopted for correcting the distance between the nodes in (4). Localization accuracy of algorithm depends on the value of the weight between unknown node and anchor node.

$$
\left(x_{e s t}, y_{\text {est }}\right)=\left(\frac{\sum_{i=0}^{n} x_{i} w_{i}}{\sum_{i=0}^{n} w_{i}}, \frac{\sum_{i=0}^{n} y_{i} w_{i}}{\sum_{i=0}^{n} w_{i}}\right)
$$

where $w_{i}$ refers to the weight from the unknown node to different anchor nodes.

\subsection{Bat Algorithm}

Bat algorithm (BA) [13] is a new metaheuristic optimization algorithm proposed by Xin-She Yang from the University of Cambridge in 2010 for searching globally optimal solution. Simulating the echo location behavior of bats, bat algorithm searches globally for optimal solution through different pulse emission rate and loudness. In the simulation of bat's searching, the formula of the position $x_{i}^{t}$ of bat at the time of $t$, the velocity $v_{i}^{t}$ and frequency $f_{i}$ is as below:

$$
\begin{gathered}
f_{i}=f_{\text {min }}+\left(f_{\text {max }}-f_{\text {min }}\right) \beta \\
v_{i}^{t}=v_{i}^{t-1}+\left(x_{i}^{t-1}-x^{*}\right) f \\
x_{i}^{t}=x_{i}^{t-1}+v_{i}^{t}
\end{gathered}
$$

In (5), $\beta \in[0,1]$ is a random vector subject to even distribution. In (6), $x^{*}$ stands for the current globally optimal solution obtained from comparing the solutions searched by $n$ bats. For local searching, if one solution is selected from the current optimal solutions, then each bat will generate local new solution as per the random walk method:

$$
x_{\text {new }}=x_{\text {old }}+\varepsilon A^{t}
$$


In (8), $\varepsilon \in[-1,1]$ is a random number subject to even distribution, $A^{t}$ refers to the average volume of all bats at the same period, $x_{\text {old }}$ is the current solution and $x_{\text {new }}$ is the new local solution.

In this paper, we fit curve from RSS Gaussian probability density function (PDF) to triangular membership function bat algorithm. Assume that $Y_{i}$ stands for RSS Gaussian PDF under real environment and fixed space, $y_{i}$ stands for triangle membership function, and then the fitness function defining bat algorithm is:

$$
f_{\text {fitness }}(k)=\sum_{i=0}^{n}\left(Y_{i}-y_{i}\right)^{2}
$$

Where $k$ refers to the slope of $y$ curve of the triangle membership function, $n$ stands for the number of RSS values. The main steps of bat algorithm are as below:

Step 1: Set initial parameters: population quantity $n$, loudness $A_{i}$, pulse emission rate, iterations and boundary conditions;

Step 2: Generate random initial population, initialize the position of the bat population $x_{i}(i=1,2,3, \ldots, n)$ and velocity $v_{i}$;

Step 3: Generate new solution by adjusting frequency;

Step 4: Update velocity and position based on (5)-(7);

Step 5: Select one from the optimal solution collection and generate a local solution surrounding the selected optimal solution;

Step 6: Obtain a new solution through random fly;

Step 7: Rank the bats and get the current optimal;

Step 8: Progressively increase cycle iteration and repeat iteration from step 3 to step 8;

Step 9: Meet the end condition and reach the maximum iterations.

\section{Fuzzy Inference System}

FIS is widely applied in uncertain and nonlinear systems. Obtaining weight through FIS is of higher environmental disturbance robustness. In weighted centroid algorithm, weight selection is an importance factor in system design. Affected by indoor environment, received RSS values are unstable, RSS value measured at the same distance may vary, and thus higher localization error is caused. As the obtained RSS is not a single value but a range, to distinguish RSS value with critical dichotomy, larger error will be easily caused. FIS is a type of intelligent control based on the rule of "If-Then", featuring excellent robustness for nonlinear control, and it can favorably restrain environment variable's impact on weighted value. Calculating weighted value through FIS can get better localization accuracy. FIS includes two methods which are Mamdani and Sugeno fuzzy system. Of the two, Mamdani is the most commonly seen. In the paper, Mamdani fuzzy system has been adopted. Principle of calculating centroid coordinates through Mamdani fuzzy system is as shown in Figure 2.

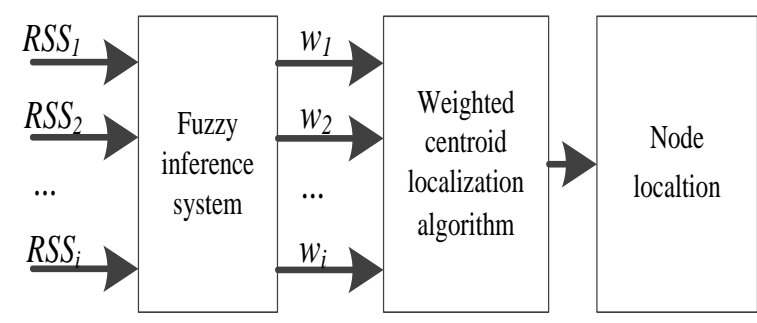

Figure 2. Weighted Centroid Localization Algorithm Based on FIS 


\subsection{Input Membership Function}

In [11], the RSS range evenly divide into 9 zones and define as nine triangle membership functions which are VVL, VL, L, ML, M, MH, H, VH and VVH as shown in Figure 3.

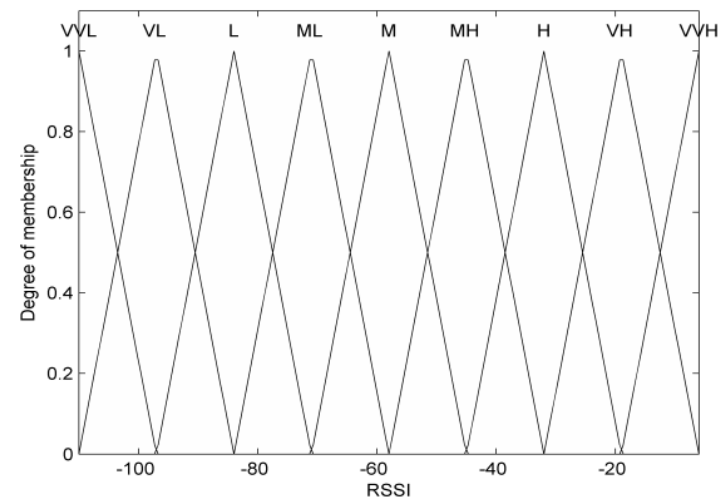

Figure 3. Input Membership Function with Isometric RSS

The membership function is the application basis for fuzzy control, and correct construction of membership function is one of the keys for fuzzy control. However, due to the complicated environment of the indoors, RSS and distance are not of linear relation. Certain errors will be encountered when calculating centroid based on the membership function.

In (2), random variable $X_{p}$ is subject to a zero-mean Gaussian white noise process and the RSS value between the same node spaces is subject to the Gaussian probability density function. In the real indoors, select the spaces of nodes of 1, 3, 5, 7 and 9 meters, and measure the RSS values respectively for 1000 times. The average values and standard deviations of RSS at different spaces of the nodes are as shown in Table 1.

Table 1. Mean and Standard Deviations of RSS at Different Spaces

\begin{tabular}{ccc}
\hline $\begin{array}{c}\text { Distance } \\
/ \mathrm{m}\end{array}$ & $\begin{array}{c}\text { RSS mean } \\
/ \mathrm{dBm}\end{array}$ & $\begin{array}{c}\text { RSS standard deviation } \\
/ \mathrm{m}\end{array}$ \\
\hline 1 & -40.78 & 0.9705 \\
3 & -45.19 & 0.8921 \\
5 & -50.49 & 1.5289 \\
7 & -54.35 & 1.8435 \\
9 & -56.50 & 2.1355 \\
\hline
\end{tabular}

Respectively put the RSS average values and standard deviations in Table 1 into the Gaussian probability density function and get the Gaussian probability density function of RSS at different spaces. It is assumed as VF(very far), $\mathrm{F}($ far), M(medium), N(near), $\mathrm{VN}$ (very near) as shown in Figure 4. 


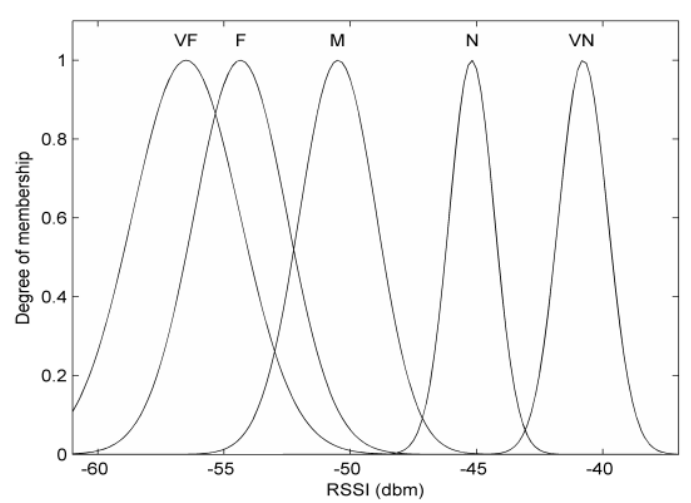

Figure 4. RSS Gaussian Probability-Density Function at Different Distances

Through bat algorithm, fit the Gaussian probability density function in Figure 4 into triangle membership function. The triangle membership function curve is as shown in Figure 5.

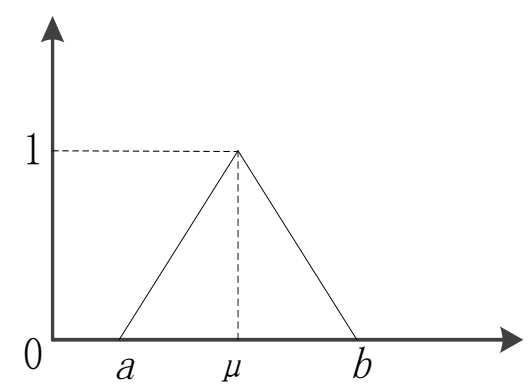

Figure 5. Triangle Membership Function

Description of triangle membership function is as below:

$$
y(x)= \begin{cases}\frac{x-a}{\mu-a}, \text { if } & a \leq x<\mu \\ \frac{b-a}{b-\mu}, \text { if } & \mu \leq x<b \\ 0, & \text { Other }\end{cases}
$$

Assume the linear function of the triangle membership function is:

$$
y(x)=k(x-\mu)+1
$$

Where $k$ stands for the slope of the rectilinear. Assume that $Y_{i}$ represents the RSS Gaussian probability density function, $y_{i}$ stands for the value of the triangle membership function and the error $e_{i}=Y_{i}-y_{i}$, then the overall error $E$ at the number of $n$ is as below:

$$
E=\sum_{i=0}^{n} e_{i}^{2}=\sum_{i=0}^{n}\left(Y_{i}-k\left(x_{i}-\mu\right)-1\right)^{2}
$$

Fitness function of bat algorithm is given by:

$$
f_{\text {fitness }}(k)=\sum_{i=0}^{n}\left(\frac{1}{\sqrt{2 \pi} \sigma} e^{-\frac{\left(x_{i}-\mu\right)^{2}}{2 \sigma^{2}}}-k\left(x_{i}-\mu\right)-1\right)^{2}
$$


where $\mu$ and $\sigma$ are the average value and standard deviation of RSS, put the $\mathrm{n}$ and $x_{i}$ into (13) and use bat algorithm to get the optimal solution of the slope $k$. Parameters of different spaces of $a$ and $b$ are as shown in Table 2 .

Table 2. Triangle Membership Function Parameters of Different Spaces

\begin{tabular}{cccccc}
\hline & VF & $\mathrm{F}$ & $\mathrm{M}$ & $\mathrm{N}$ & $\mathrm{VN}$ \\
\hline$a$ & $/$ & -59.22 & -54.21 & -49.25 & -43.43 \\
$b$ & -51.04 & -49.25 & -46.56 & -42.67 & $/$ \\
\hline
\end{tabular}

Respectively put the values of $a$ and $b$ in Table 2 into (11) to get the triangle membership function as shown in Figure 6.

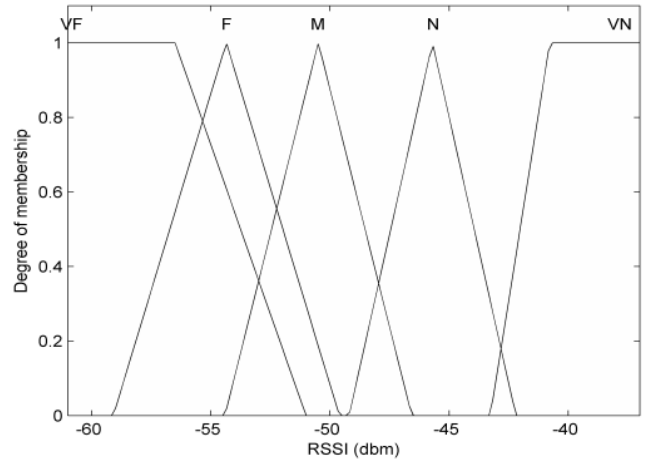

Figure 6. Triangle Membership Function of RSS in Real Environment

\subsection{Output Membership Function}

Output membership function of FIS is the weight between unknown node and anchor node. The RSS value maps into weigh with range in $[0,1]$. Output membership function is defined as five linear functions which are VL (very little), L (little), M (middle), $\mathrm{H}$ (high), VH (very high) as shown in Figure 7.

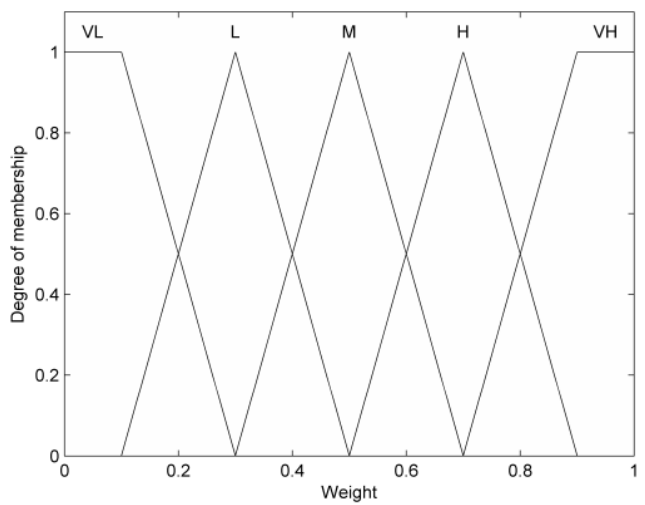

Figure 7. Output Membership Function

\subsection{Fuzzy Rules}

Based on the input variable RSS value and output weighted value, design the fuzzy rule of the fuzzy system. If the RSS value received is high, then it indicates that the node is near the anchor node; and vice versa. Fuzzy rule is as shown in Table 3. 


\section{Table 3. Fuzzy Rules}

\begin{tabular}{lcc}
\hline Rule list & RSS & Weight \\
\hline Rule 1 & VF & VL \\
Rule 2 & F & L \\
Rule 3 & M & M \\
Rule 4 & N & H \\
Rule 5 & VN & VH \\
\hline
\end{tabular}

In accordance with the fuzzy rules, generate the surface curve of input and output in FIS as shown in Figure 8.

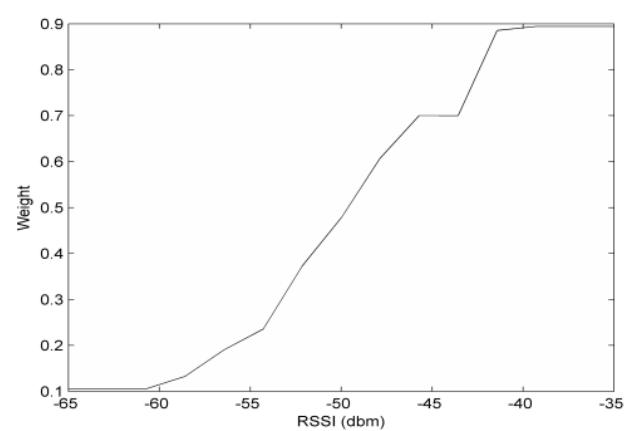

Figure 8. Weight Corresponding to RSS Input

\subsection{Fuzzy Inference Process}

The paper has adopted Mamdani fuzzy system and the rule is as below:

$$
\text { Rule: If RSS is X Then Weight is } Z
$$

To illustrate the calculation process of FIS, take the input of RSS $=-47.5 \mathrm{dBm}$ as the example. From Figure 6, RSS value can be obtained as below:

$$
\begin{aligned}
& X_{M}(-47.5)=0.2372 \\
& X_{N}(-47.5)=0.485
\end{aligned}
$$

According to Mamdani theory:

$$
Z^{\prime}(W)=\max [X(R S S) \wedge Z(W)]
$$

The processes as shown in Table 4 .

Table 4. Mamdani Fuzzy Inference Result

\begin{tabular}{ccc}
\hline$X(R S S)$ & $X_{M}(-47.5)=0.2372$ & $X_{N}(-47.5)=0.4857$ \\
\hline$Z^{\prime}(W)$ & $0.2372 \Lambda Z_{M}$ & $0.4857 \Lambda Z_{H}$ \\
\hline
\end{tabular}

According to the fuzzy rules, the output result is as shown in Figure 9. 


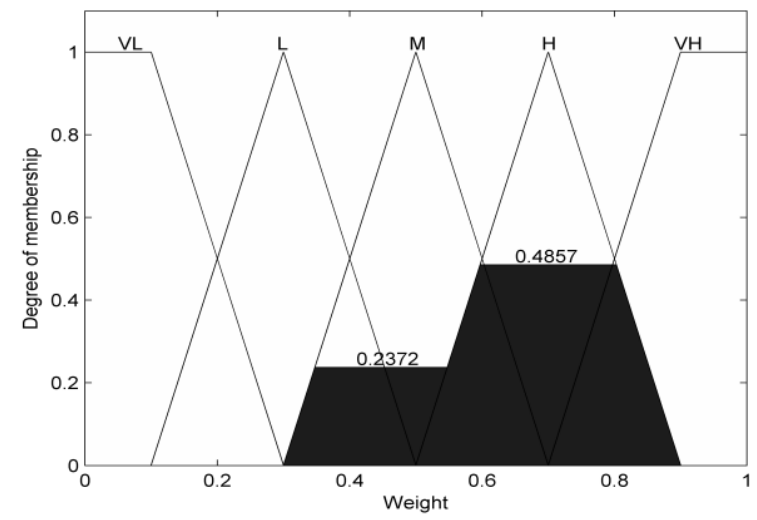

Figure 9. Fuzzy System Output Results

FIS calculates the results based on multiple rules and each rule will give a result, therefore, fuzzy system conclusion is a fuzzy collection which should go through ambiguity resolution for getting real values. Ambiguity solution can be realized through various methods including center-of-gravity method, maximum median method and maximum mean value method [14].Center-of-gravity method for ambiguity solution is of high algorithm accuracy and can get more precise value; therefore, it gains its popularity [15]. In the paper, center-of-gravity method has been adopted. The principle is as below: to get the center of gravity of an area, assume the area as a plate of equal density and the centroid obtained will balance it [16]. The formula is as below:

$$
d=\frac{\int y(x) x d x}{\int y(x) d x}
$$

The membership value is the algebraic integral in continuous domain. Based on (18), the center of gravity of the above dash area can be obtained: $w=0.633$.

\section{Experiment and Analysis}

\subsection{Experimental Environment Setting}

To verify the accuracy of the algorithm, I have experimented in real indoor environment. The experiment has been set in an indoor plane floor of $10 \mathrm{~m} \times 10 \mathrm{~m}$ with anchor nodes set at $\mathrm{A}(0,0), \mathrm{B}(10,0), \mathrm{C}(0,10)$ and $\mathrm{D}(10,10)$ as shown in Figure 10.

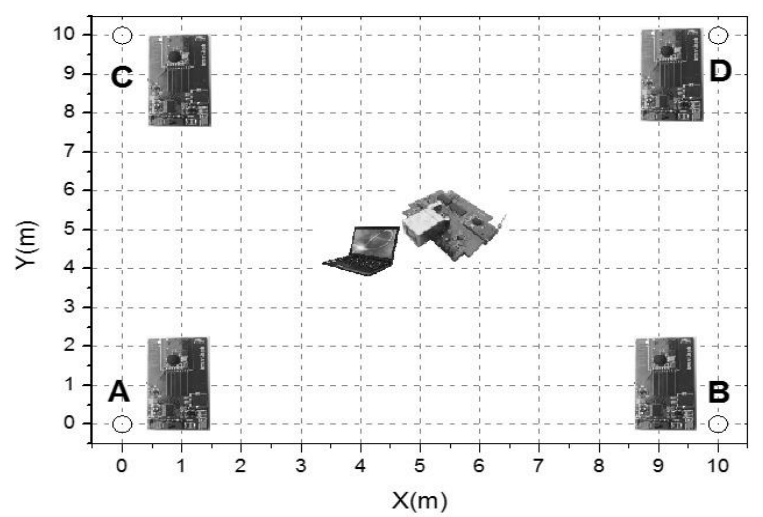

Figure 10. Sketch Map of the Experiment Area 


\subsection{Hardware of Localization System}

Wireless sensor adopts the equipment prepared by the author. The chip includes TI MSP430G2452 microprocessor and TI CC2500 low-power is $2.5 \mathrm{G}$ radio frequency chip with wave strip antenna. The maximum transmitted power of CC2500 is $0 \mathrm{dBm}(1 \mathrm{~mW})$. Under $2.4 \mathrm{kbps}$ data rate, the sensitivity of the receiving terminal is $-108 \mathrm{dBm}$. The wireless sensor can reach 50 meters indoors, easy constructing indoor experiments. The signal hub connect to PC through USB and receive RSS data of the unknown nodes. The FIS on the PC will run and get the weighted value between the sensor nodes and the anchor nodes, and lastly get the centroid through weighted centroid calculation formula.

\subsection{Average Localization Error}

The localization error between the real localization and estimation localization of the node is calculated as below:

$$
\text { Localization error }=\sqrt{\left(x_{e s t}-x_{r}\right)^{2}+\left(y_{e s t}-y_{r}\right)^{2}}
$$

For all the unknown nodes, obtain the average localization error by

$$
\text { Average localization error }=\frac{\sum \sqrt{\left(x_{e s t}-x_{r}\right)^{2}+\left(y_{e s t}-y_{r}\right)^{2}}}{n}
$$

Where $n$ is the number of unknown nodes, $\left(x_{e s t}, y_{e s t}\right)$ is the estimated centroid localization while $\left(x_{r}, y_{r}\right)$ is the real centroid localization.

\subsection{Experiment and Analysis}

Experiment on the Zigbee platform, the sensor nodes are placed on the area of $9 \mathrm{~m} \times 9 \mathrm{~m}$ with the interval of one meter and use three centroid localization algorithms for calculation. The first algorithm is based on the (3) is called CA. The second algorithm is weighted centroid localization algorithm based on Mamdani fuzzy inference with Figure 3 membership function is called FCA. The last algorithm is weighted centroid localization algorithm based on Mamdani fuzzy inference with membership function (Figure 6) optimized by bat algorithm is called MFCA.

In the experiment, the sensor node simulation result and localization error estimated by CA are as shown in Figure 11. The left figure represents sensor node distribution, while the right figure represents error bar of each sensor node. On the left figure, the solid dots at the four corners stand for four anchor nodes, stars represent 81 sensor nodes and the hollow circles are the theoretical centroids estimated, while the solid lines refer to the localization error between the theoretical and real centroids. On the right figure, the abscissas stand for the number of the measured sensor nodes while the ordinates refer to the localization errors.
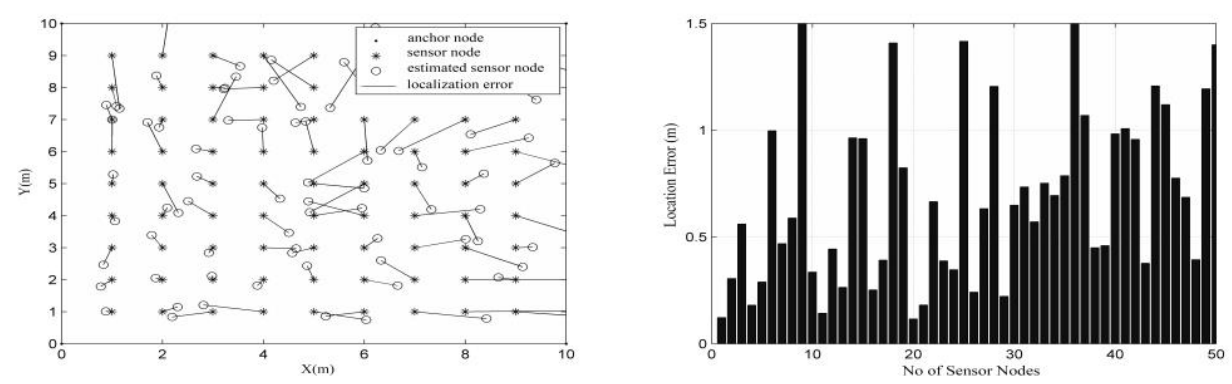

Figure 11. Simulation Result of Localization and Localization Error by CA 
In the experiment, the sensor node simulation result and localization error estimated by FCA are as shown in Figure 12.
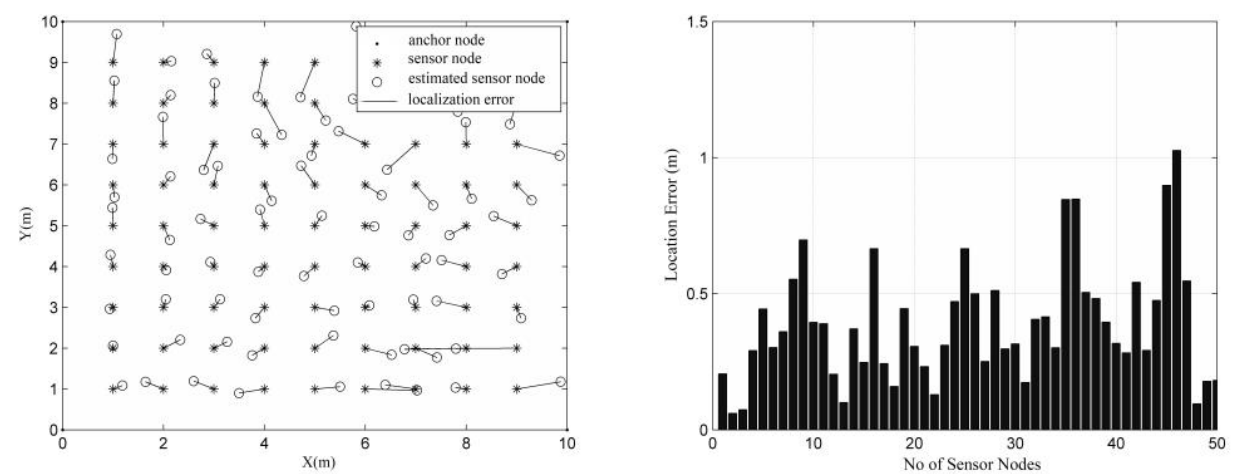

Figure 12. Simulation Result of Localization and Localization Error by FCA

In the experiment, the sensor node simulation result and localization error estimated by MFCA are as shown in Figure 13.
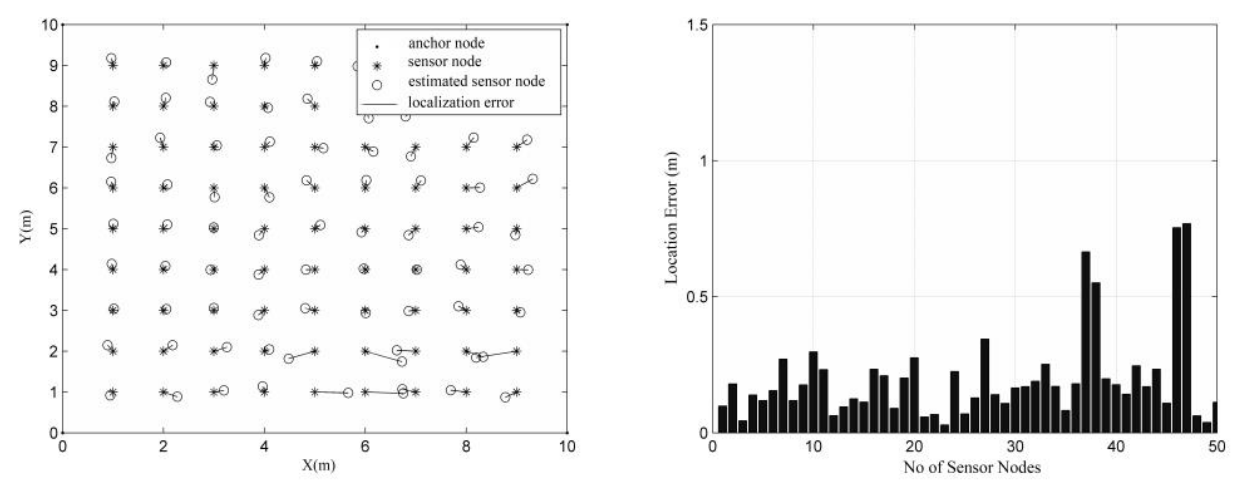

Figure 13. Simulation Result of Localization and Localization Error by MFCA

In accordance with (20) and calculate the average localization error of the different methods as shown in Table 5 .

Table 5. Average Localization Error

\begin{tabular}{cccc}
\hline Algorithm & $\begin{array}{c}\text { Min } \\
\text { error/m }\end{array}$ & $\begin{array}{c}\text { Max } \\
\text { error/m }\end{array}$ & $\begin{array}{c}\text { Mean } \\
\text { error/m }\end{array}$ \\
\hline CA & 0.1451 & 1.3036 & 0.8990 \\
FCA & 0.0251 & 0.9942 & 0.4187 \\
MFCA & 0.0158 & 0.3931 & 0.2156 \\
\hline
\end{tabular}

As shown in Table 5, the average localization accuracy of MFCA is $76.02 \%$ higher than that of CA and $53.42 \%$ higher than that of FCA, indicating that the optimized membership function makes weighted value more accurate and the localization error smaller.

Cumulative distribution function (CDF) is the integration of probability density function and describes the cumulative probability distribution of random variables. It is designed as below:

$$
F(x)=P(X \leq x)
$$


Use CDF to analyze the localization errors and compare the cumulative distribution functions of the localization errors with different algorithms as shown in Figure 14.

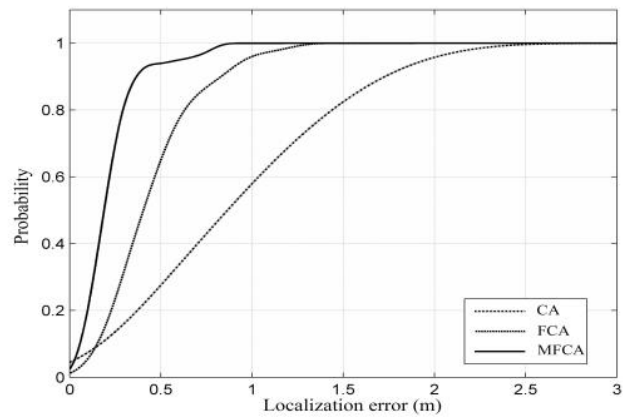

\section{Figure 14. Cumulative Distribution Functions of Localization Error}

Figure 14 indicates that $90 \%$ of the localization error of CA is at $1.63 \mathrm{~m}, 90 \%$ of that of FCA is at $0.78 \mathrm{~m}$ and $90 \%$ of that of MFCA is at $0.32 \mathrm{~m}$. MFCA improves the localization accuracy because of membership function optimized by bat algorithm.

\section{Conclusions}

In real environment, because of the radio interference arises by many factors, so the existing algorithms, such as ToA, TDoA, AoA, RSS and other location algorithms will produce large error. Range-free localization is widely applied for its simple realization and for it requiring no complicated hardware. The paper has modified the weighted localization algorithm base on FIS. The RSS membership function optimized by bat algorithm is adopted in fuzzy system to output more precise weighted value, overcoming the RSS drift effect in interior localization application which causes imprecise localization, and reducing the indoor environmental impact on RSS. The experiment results indicate that the system is of simple realization and low cost. It is applicable to localization in complicated indoor environment, and is able to meet indoor localization service.

\section{Acknowledgements}

The research work was supported by National Science \& Technology Pillar Program during the 12th Five-year Plan Period under Grant No. 2012BAD10B01.

\section{References}

[1] D. F. Larios, J. Barbancho, F. J. Molina and C. Leon, "LIS: Localization based on an intelligent distributed fuzzy system applied to WSN", Ad Hoc Networks, vol. 5, no. 10, (2012), pp. 604-622.

[2] D. P. Qiao and G. K. H. Pang, "Evolutionary approach on connectivity-based sensor network localization", Applied Soft Computing, vol. 22, (2014), pp. 36-46.

[3] S. S. Wang, K. P. Shih and C. Y. Chang, "Distributed direction-based localization in wireless sensor networks", Computer Communications, vol. 30, no. 6, (2007), pp. 1424-1439.

[4] C. C. Chen and T. C. Lin, "A Low-Cost Anchor Placement Strategy for Range-Free Localization Problems in Wireless Sensor Networks", International Journal of Distributed Sensor Networks, vol. 2, no. 3, (2013), pp. 23-30.

[5] L. Q. Gui, V. Thierry and A. Wei, "Improvement of range-free localization technology by a novel DVhop protocol in wireless sensor networks", Ad Hoc Networks, vol. 8, (2014), pp. 1-19.

[6] F. C. Xu and Z. Liu, "A New Node Self-Localization Algorithm Based RSSI for Wireless Sensor Networks", Proceedings of the Fifth Computational and Information Sciences (ICCIS), (2013) pp. 1616 $-1619$.

[7] M. Shon, J. Minho and H. Choo, "An interactive cluster-based MDS localization scheme for multimedia information in wireless sensor networks", Computer Communications, vol. 35, no. 15, (2012), pp.19211929. 
[8] H. H. Wang, J. C. Wan and R. Y. Liu, “A Novel Ranging Method Based on RSSI”, Energy Procedia, vol. 12, (2011), pp. 230-235.

[9] M. D. Li, W. Xiong and L. Guo, "Improvement of DV-Hop Localization Algorithm Based on Artificial Bee Colony Algorithm", Computer Science, vol. 40, no. 1, (2013), pp. 149-152.

[10] D. S. Han, W. Yang, Y. Liu and Y. Liu and Y. Zhang, "A Weighted Centroid Localization Algorithm Based on Received Signal Strength indicator for Underground Coal Mine", Journal of China Coal Society, vol. 3, no. 38, (2013), pp. 522-528.

[11] A. Kumar, N. Chand and V. Kumar, "Range Free Localization Schemes for Wireless Sensor Networks", International Journal of Computer Networks \& Communications (IJCNC), vol. 3, no. 6, (2011), pp. 132144.

[12] J. Proakis and M. Salehi. "Digital Communications", New York, McGraw-Hill Press, (2010), pp. 165170.

[13] X. S. Yang, "A New Metaheuristic Bat-Inspired Algorithm: Nature Inspired Cooperative Strategies for Optimization (NISCO 2010)", Studies in Computational Intelligence, Springer Berlin, (2010), pp. 65-74.

[14] T. Skulavik, M. Kopcek and P. Mydlo, "The defuzzification methods influence on fuzzy control of nuclear reactor", Proceeding of International Symposium on Computational and Business Intelligence (ISCBI 2013), New Delhi: IEEE Press, (2013), pp. 119-122.

[15] T. Mitsuishi, "Continuity of Approximate Reasoning Using Center of Sums Defuzzification Method", Proceedings of the 35th International Convention on Information and Communication Technology, Electronics and Microelectronics (MIPRO), Opatija: IEEE Press, (2013), pp. 991-994.

[16] B. C. Arabacioglu, "Using fuzzy inference system for architectural space analysis", Applied Soft Computing, vol. 10, no. 3, (2009), pp. 926-937.

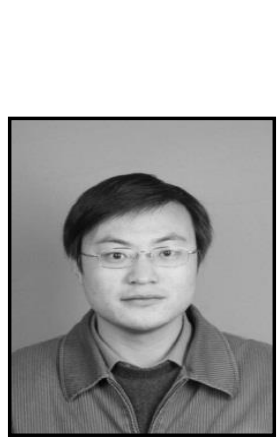

\section{Authors}

Hao Shi, he received M.S. degree in Computer Science in 2002 and $\mathrm{Ph} . \mathrm{D}$. candidate in Control Theory and Control Engineering of Zhejiang University of Technology, China. His research interest covers WSN technology and intelligent algorithm.

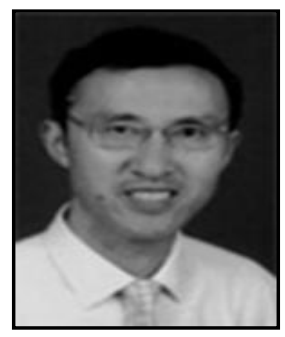

Wanliang Wang, he received Ph.D. degree in Control Theory and Control Engineering in 2001. He has devoted nearly 20 years to educational work and now he is the dean of School of Computer Science and Technology at the Zhejiang University of Technology. As a researcher, he is leading a large research group in the field of simulation for small hydropower projects and many valuable achievements have made. His research interest covers intelligent algorithms and network control. 
International Journal of Future Generation Communication and Networking Vol. 9, No. 5 (2016) 